\title{
Incremental Net Monetary Benefit of Bariatric Surgery: Systematic Review and Meta-Analysis of Cost-Effectiveness Evidences
}

\author{
Prapaporn Noparatayaporn ${ }^{1,2} \cdot$ Montarat Thavorncharoensap $^{1,3}$ (D) Usa Chaikledkaew ${ }^{1,3}$. \\ Bhavani Shankara Bagepally ${ }^{4}$. Ammarin Thakkinstian ${ }^{1,5}$
}

Received: 18 August 2020 / Revised: 3 April 2021 / Accepted: 7 April 2021 / Published online: 24 April 2021

(C) The Author(s) 2021

\begin{abstract}
This systematic review aimed to comprehensively synthesize cost-effectiveness evidences of bariatric surgery by pooling incremental net monetary benefits (INB). Twenty-eight full economic evaluation studies comparing bariatric surgery with usual care were identified from five databases. In high-income countries (HICs), bariatric surgery was cost-effective among mixed obesity group (i.e., obesity with/without diabetes) over a 10-year time horizon (pooled INB $=\$ 53,063.69 ; 95 \%$ CI $\$ 42,647.96$, $\$ 63,479.43$ ) and lifetime horizon (pooled INB $=\$ 101,897.96 ; 95 \%$ CI \$79,390.93, \$124,404.99). All studies conducted among obese with diabetes reported that bariatric surgery was cost-effective. Also, the pooled INB for obesity with diabetes group over lifetime horizon in HICs was $\$ 80,826.28$ (95\% CI $\$ 32,500.75, \$ 129,151.81$ ). Nevertheless, no evidence is available in low- and middle-income countries.
\end{abstract}

Keywords Incremental net monetary benefit $\cdot$ Cost-effectiveness $\cdot$ Bariatric surgery $\cdot$ Economic evaluation

\section{Introduction}

Bariatric surgery is an attractive treatment option for obese patients, who could not achieve weight control by conservative, non-surgical therapies. The clinical effectiveness of bariatric surgery has been well established. Evidences from previous systematic reviews consistently indicated that bariatric surgery could significantly reduce body weight and improve comorbidities, as compared with usual care (e.g., pharmacotherapy and/or lifestyle modification) [1-5]. In addition,

Key points:

- Bariatric surgery seems to be cost-effective over lifetime horizon in high-income countries (HICs).

- The pooled INB for bariatric surgery as compared with usual care in HICs was between $\$ 81,000$ and $\$ 102,000$.

- No evidence is available in low- and middle-income countries.

Montarat Thavorncharoensap

montarat.tha@mahidol.ac.th

1 Mahidol University Health Technology Assessment (MUHTA) Graduate Program, Bangkok, Thailand

2 Pharmacy Department, Faculty of Medicine Siriraj Hospital, Mahidol University, Bangkok, Thailand bariatric surgery might be superior to usual care for shortterm remission of diabetes mellitus $[1,6]$. Nevertheless, with the growing demand for bariatric surgery, demonstrating only the clinical effectiveness is not sufficient given the limited resources for healthcare. Evidences on the value for money are strongly required to support policy decision-making. To date, there were many published systematic reviews and metaanalyses on bariatric surgery [7-9]. However, most studies were limited to the description of methodologies and economic evaluation results [9]. Existing quantitative synthesis
3 Social and Administrative Pharmacy Division, Department of Pharmacy, Faculty of Pharmacy, Mahidol University, 447 Sri Ayudhaya Rd., Rajathevi, Bangkok 10400, Thailand

4 ICMR-National Institute of Epidemiology, Chennai, India

5 Department of Clinical Epidemiology and Biostatistics, Faculty of Medicine Ramathibodi Hospital, Mahidol University, Bangkok, Thailand 
pertained only to differences in healthcare cost pre- and postsurgery or between surgical and non-surgical approaches [7, 8]. Consequently, determining whether bariatric surgery provides value for money or in which condition it might be costeffective is still controversial. Therefore, a meta-analysis which pools the value for money of bariatric surgery is required.

Most economic evaluation studies report incremental costeffectiveness ratios (ICERs), which represent the ratio of incremental $\operatorname{cost}(\Delta C)$ between the two interventions and incremental effectiveness $(\Delta \mathrm{E})$ between the same groups $[10,11]$. The effectiveness (E) is usually measured in terms of amount of quality-adjusted life year (QALY) gained or disabilityadjusted life year (DALY) averted. The ICER is then compared with the pre-defined cost-effectiveness threshold (K), the maximum amount a decision-maker is willing to pay for one QALY or DALY (e.g., $£ 20,000$ per QALY in the UK [12], or one time the GDP per capita per DALY in several countries [13]). If the ICER is less than K, the intervention is cost-effective; otherwise, it is not cost-effective. Nevertheless, the interpretation of ICER is problematic when its value is negative, which may indicate a lower cost with higher effectiveness or higher cost along with lower effectiveness of interventions. Thus, there is ambiguity in interpretation [10].

The incremental net monetary benefit (INB), which is the difference in net monetary benefit between the new intervention and the standard intervention [10], has been recently used instead of the ICER. The INB can be computed as the difference of incremental monetary benefit and incremental cost $(\mathrm{INB}=(\Delta \mathrm{E} \times \mathrm{K})-\Delta \mathrm{C})[10,11]$. It is relatively easier to interpret than the ICER. A positive INB (INB $>0$ ) indicates that an intervention is cost-effective as compared with the standard intervention at the given threshold, whereas a negative INB indicates the new intervention is not cost-effective relative to the standard one $[10,11,14,15]$. Up until now, few meta-analyses have been conducted to pool INBs [15-18]. Since there is still controversy on the value of money of bariatric surgery, we, therefore, conducted a meta-analysis which systematically reviewed and pooled INBs of bariatric surgery as compared with usual care among patients with obesity. Our main hypothesis is bariatric surgery is cost-effective for patients with obesity. Whenever possible, we examined whether bariatric surgery was cost-effective in particular type of patients (i.e,. obese with DM) and determined which specific procedures of bariatric surgery (i.e., SG, AGB, RYGB) were cost-effective when compared with usual care.

\section{Materials and Methods}

The protocol of this systematic review was conducted and reported according to the Preferred Reporting Items for Systematic Reviews and Meta-Analyses (PRISMA) [19] (see Table S1). The review protocol was registered at the PROSPERO (registration number CRD42019142147).

\section{Data Sources and Search Strategies}

We searched relevant studies from PubMed, Scopus, the Cochrane Central Register of Controlled Trials-Wiley library, the Cost-Effective Analysis (CEA) Registry (The CEA Registry), and the Centre for Reviews and Dissemination (CRD) since inception to July 2019 without language restrictions. We constructed search terms based on the population, intervention, outcome, and study design domains (i.e., obesity, bariatric surgery, incremental cost-effectiveness ratio, and economic evaluation) (see Table S2).

\section{Study Selection}

Two reviewers (P.N. and M.T.) independently selected studies by screening titles and abstracts.Full texts were then reviewed based on the following criteria: any full economic evaluation study (i.e., cost-effectiveness analysis (CEA), cost-utility analysis (CUA), cost-benefit analysis (CBA)) of adult obesity (i.e., $\mathrm{BMI}>32 \mathrm{~kg} / \mathrm{m}^{2}$ ); compared any pair of bariatric surgery (e.g., open or laparoscopic surgeries of adjustable gastric banding (AGB), Roux-en-Y gastric bypass (RYGB), sleeve gastrectomy (SG), and a mix of these bariatric surgeries (BS)) with usual care (e.g., pharmacotherapy and/or lifestyle modification); provided sufficient data for calculating INB [17]. The studies were excluded if they studied patients with other specific diseases or their full texts were not available.

\section{Data Extraction}

Data were extracted by two independent reviewers (P.N. and B.S.). A standardized data extraction form was developed. The data required for estimating INB were extracted: cost $(C)$, incremental cost $(\Delta C)$, effectiveness (E), incremental effectiveness $(\Delta \mathrm{E})$, and ICER. These parameter values were extracted as means and dispersions (i.e., SDs, SEs, and 95\% CIs). In some cases, $\Delta \mathrm{C}$ and $\Delta \mathrm{E}$ data were extracted from probabilistic sensitivity analyses (PSA). Authors of original studies were contacted further in case of incomplete information.

\section{Data Preparation}

INB was calculated as follows [10, 15]: INB $=(\mathrm{K} \times \Delta \mathrm{E})-$ $\Delta \mathrm{C}$ or $\Delta \mathrm{E} \times(\mathrm{K}-\mathrm{ICER})$, where $\mathrm{K}$ is the cost-effectiveness threshold, and $\Delta \mathrm{E}$ and $\Delta \mathrm{C}$ are incremental effective and incremental cost, respectively. The INB variance was estimated as follows $[15,17]$ : 
$\operatorname{var}(I N B)=K^{2} \sigma_{\Delta E}^{2}+\sigma_{I C E R}^{2}$ or $K^{2} \sigma_{\Delta E}^{2}+\sigma_{\Delta C}^{2}-2 K \rho_{\Delta C \Delta E}$, where $\sigma_{\Delta E}^{2}$ is variance of $\Delta \mathrm{E}, \sigma_{I C E R}^{2}$ is variance of ICER, $\sigma_{\Delta C}^{2}$ is variance of $\Delta \mathrm{C}$, and $\rho_{\Delta C \Delta E}$ is covariance of $\Delta \mathrm{C}$ and $\Delta \mathrm{E}$. In case of incomplete data, the variance of INB was simulated according to data availability (scenarios 1 to 5), as suggested by Bagepally et al. [17] If the study did not report the costeffectiveness threshold, the GDP per capita was used [13]. To pool data across studies, all currencies were converted to the international standard currency 2019 (international dollars; Int\$) using consumer price index (CPI) and purchasing power parity (PPP) obtained from the World Economic Outlook Database (2019) [20].

\section{Risk-of-Bias Assessment}

The quality of each study was assessed by two independent reviewers (P.N. and M.T.), using the ECOBIAS checklist [21]. Disagreement was resolved by consensus with a third reviewer (U.C.).

\section{Statistical Analysis}

The estimated INBs were pooled across studies [15-17]. To minimize heterogeneity, studies were stratified by country income level, which was classified according to the World Bank as high-income countries (HICs) and upper-middle-income countries (UMICs) [22]. In addition, the INBs were pooled according to perspective, type of model, type of patients (i.e., mixed obesity group, which included patients with/without diabetes, obesity with diabetes group), and time horizon (i.e., lifetime, 10 years). Subgroup analysis by types of bariatric surgery (i.e., AGB, RYGB, SG, BS) was also performed. A fixedeffect model by an inverse variance method was applied for pooling if heterogeneity was not present; otherwise, a random-effect model (DerSimonian and Laird) was used [23].

The heterogeneity of INBs among the studies was assessed using the Cochrane's $Q$ statistic and $I^{2}$ statistics. Publication bias was assessed using the funnel plot and Egger's test. A contour-enhanced funnel plot was applied to distinguish publication bias from other causes of asymmetry [24].

\section{Results}

\section{Study Selection}

Of the 4395 identified studies, 28 were included and analyzed (see Fig. 1). The characteristics of studies are described in Tables 1 and S3. As for country income level, 24 [25-35, $37-43,45-50](85.7 \%)$ and $4[36,44,51,52](14.3 \%)$ studies were conducted in HICs and UMICs, respectively. From these, $20(71.4 \%)$ were conducted in mixed obesity group (i.e., with/without diabetes), whereas 8 [45-52] (28.6\%) studies were conducted in obesity with diabetes group. All studies, except for one [52], were conducted in obese patients whose BMIs were $35 \mathrm{~kg} / \mathrm{m}^{2}$ and higher. The three most common interventions were AGB $(N=15,53.6 \%)$, RYGB $(N=20$, $71.4 \%)$, and SG $(N=11,39.3 \%)$.

As for methodology, all studies were CUAs. The modelbased techniques were Markov model [26, 28, 29, 33-44, 47-52] $(N=21 ; 75.0 \%)$, discrete-event simulation [30] $(N=$ $1 ; 3.6 \%)$, decision tree $[25](N=1 ; 3.6 \%)$, decision tree and lifetime natural history model [32] $(N=1 ; 3.6 \%)$, and a de novo economic model [31] $(N=1 ; 3.6 \%)$. Two studies [45, 46] (7.1\%) did not specify the type of model, while one study [27] (3.6\%) did not state the technique. Most studies $(N=25$; $89.3 \%$ ) adopted a payer perspective. One study [29] adopted societal perspective and two studies [27, 49] did not clearly mention the perspective. Lifetime, 10-year, and less than 10year horizons were respectively used in $18[25-27,29,30$, $32-35,37-43,48,49], 10$ [28, 30, 31, 34, 35, 39, 41-44], and 5 studies $[27,30,45,46,51]$.

\section{INBs of Bariatric Surgery in HICs}

The estimated INBs from HICs were presented separately by types of patients (see Tables S4 and S5). For mixed obesity group, bariatric surgery was found to be cost-effective in all except for three studies, which adopted 2-year [27], 5-year [30], and 10-year [31] time horizons. For obesity with diabetes group, all studies revealed that bariatric surgery was costeffective.

\section{Pooled INBs of Bariatric Surgery among Mixed Obesity Group in HICs}

The INBs of overall bariatric surgery versus usual care among mixed obesity group under payer perspective were highly varied across 11 studies [26, 33-35, 37-43] $\left(I^{2}=83.9 \%\right)$ with a pooled INB (95\% CI) over lifetime horizon of $\$ 101,897.96$ $(\$ 79,390.93, \$ 124,404.99)$ (see Fig. 2). Subgroup analysis indicated that $\mathrm{AGB}, \mathrm{SG}$, and mixed types of BS, but not RYGB, were significantly cost-effective as compared with usual care with pooled INBs of $\$ 51,143.29$ (95\% CI $\left.\$ 15,735.29, \$ 86,551.29 ; I^{2}=37.1 \%\right), \$ 127,578.98(95 \% \mathrm{CI}$ $\left.\$ 62,139.61, \$ 193,018.36 ; I^{2}=0 \%\right), \$ 143,438.56$ (95\% CI $\left.\$ 91,320.26, \$ 195,556.88 ; I^{2}=89.5 \%\right)$, and $\$ 110,928.33$ (95\% CI $\left.-\$ 8,677.49, \$ 230,534.14 ; I^{2}=85.2 .1 \%\right)$, respectively. A funnel plot of overall bariatric surgery effect indicated asymmetry of the funnel which contrasted to Egger's test (coefficient $=2.11$, $\mathrm{SE}=0.47, p=0.001$ ), Fig. S1. A contourenhanced funnel (Fig. S2) showed that asymmetry might be caused by heterogeneity. 


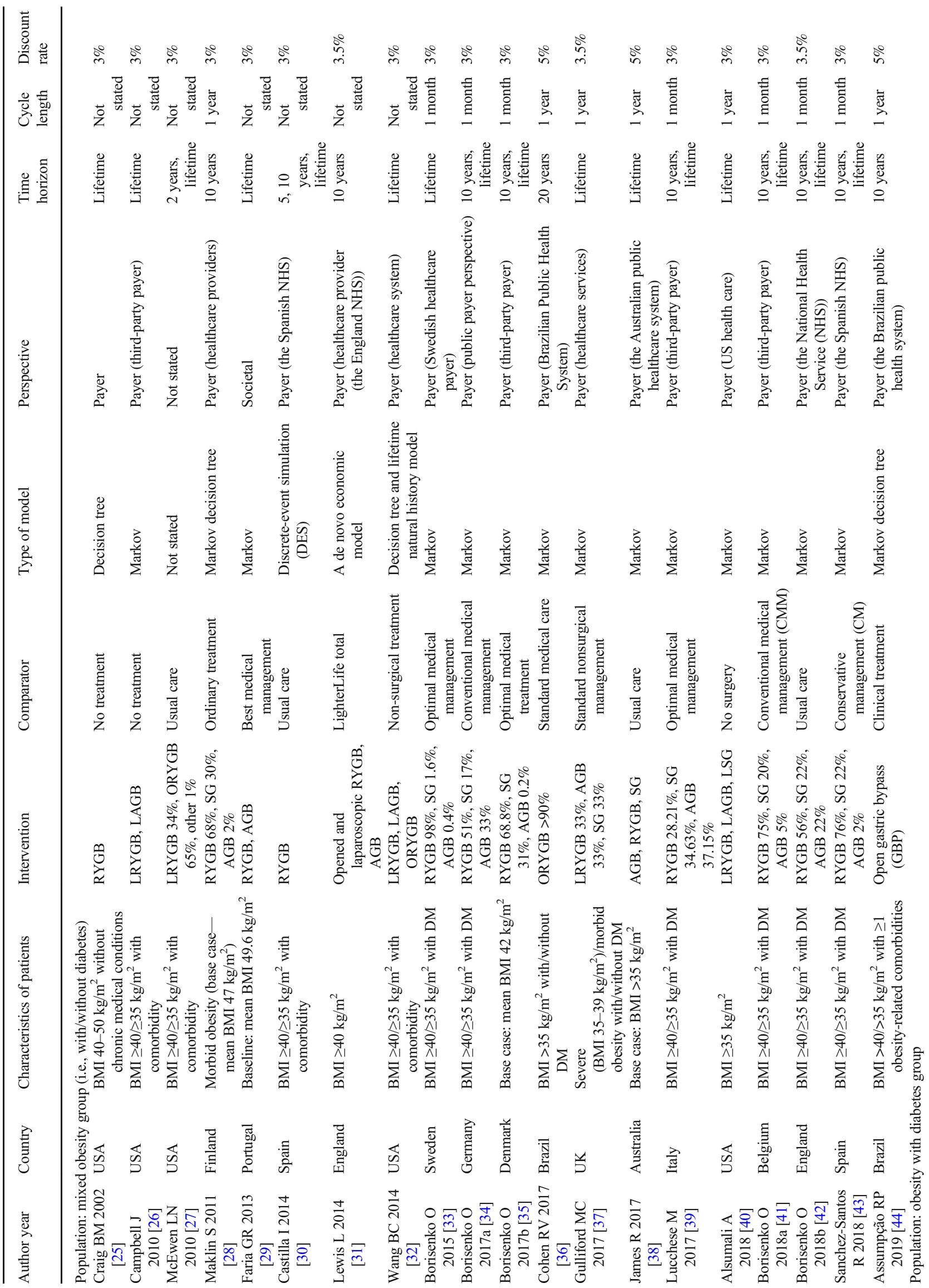




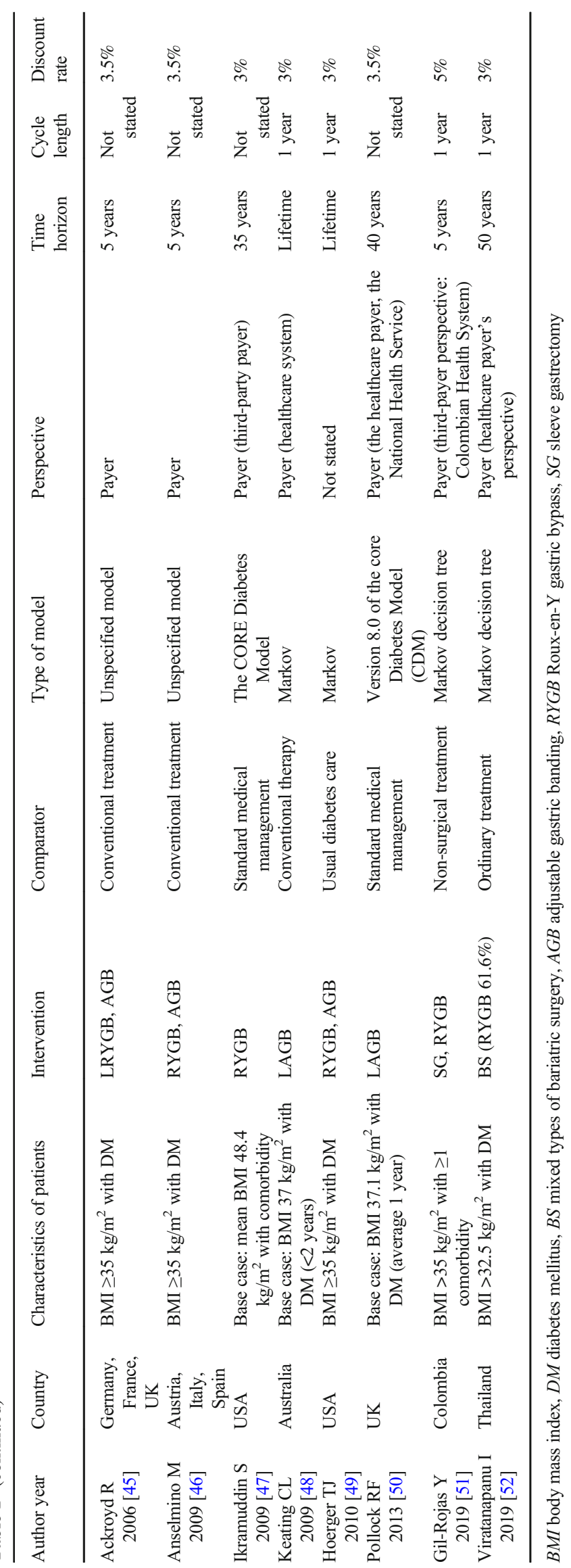




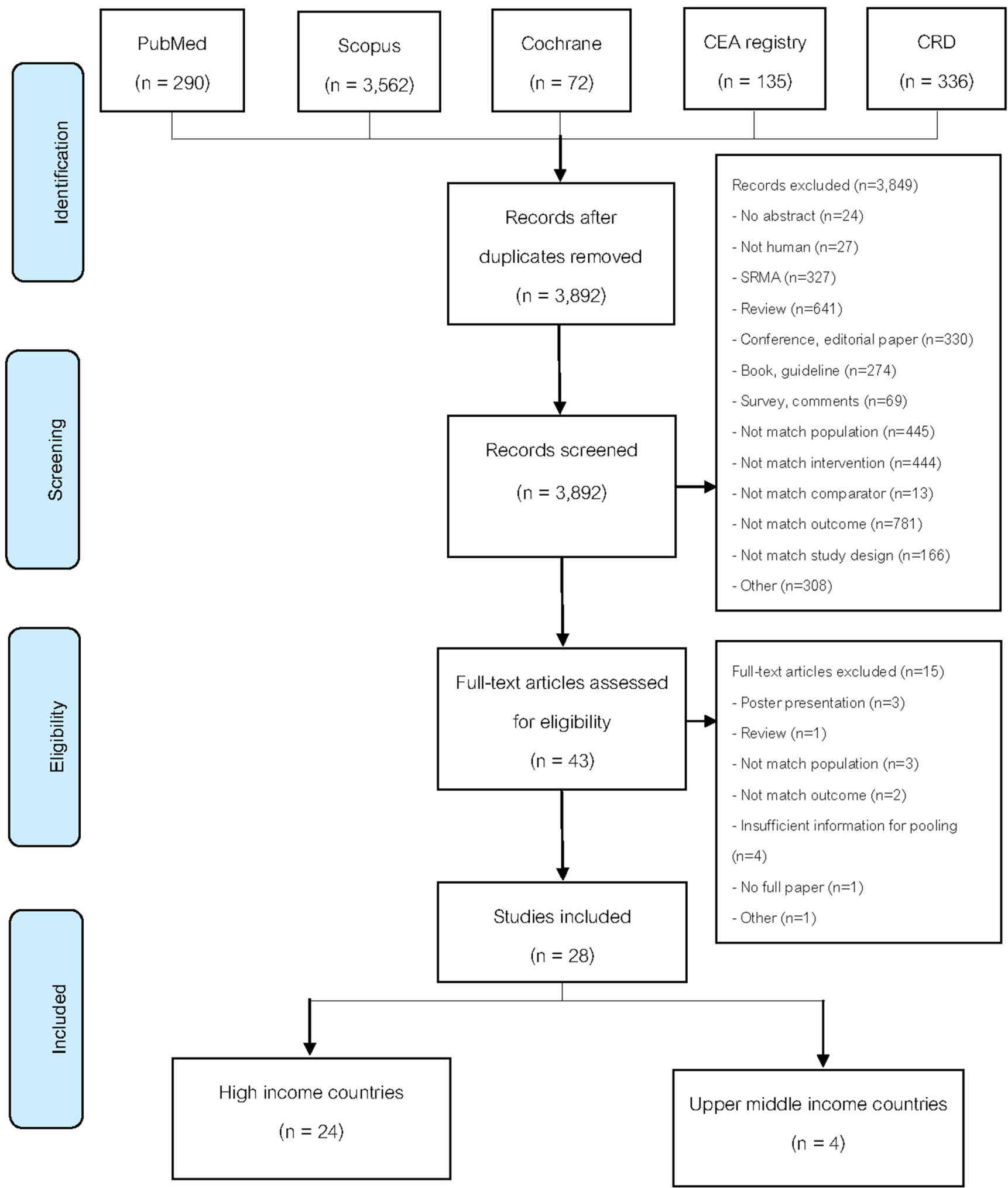

Fig. 1 Flow diagram of the included studies

The INBs of bariatric surgery among mixed obesity group over a 10-year time horizon were highly varied across seven studies $[28,34,35,39,41-43]\left(I^{2}=\right.$ $75.6 \%$ ), with a pooled INB (95\% CI) of $\$ 53,063.69$
$(\$ 42,647.96, \$ 63,479.43)$ (see Fig. 3). A funnel plot (Fig. S3) and Egger's test (coefficient $=11.13, \mathrm{SE}=$ $6.81, p=0.163$ ) indicated no evidence of publication bias. 


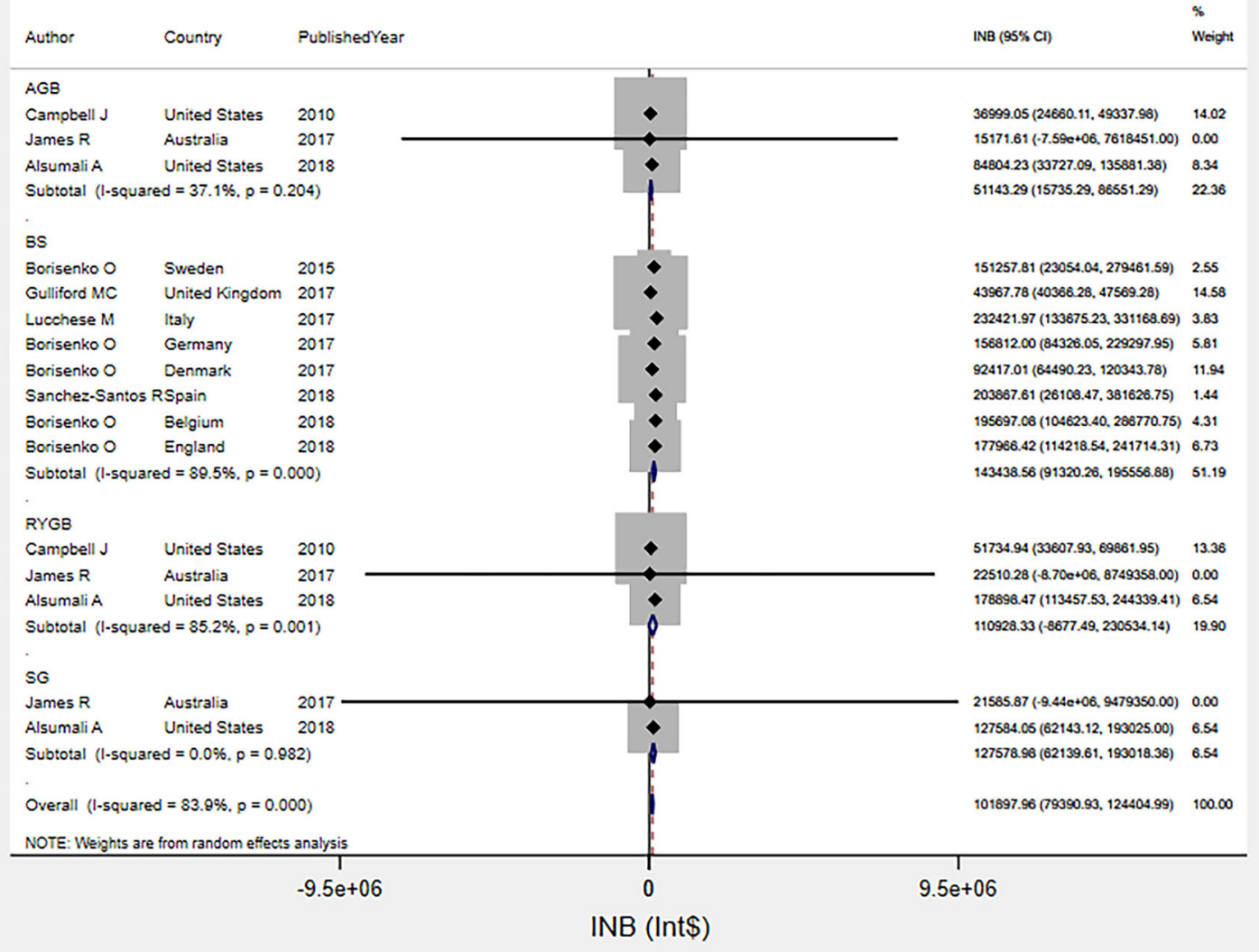

Fig. 2 Pooled INBs of bariatric surgery among mixed obesity group in high income countries over lifetime horizon

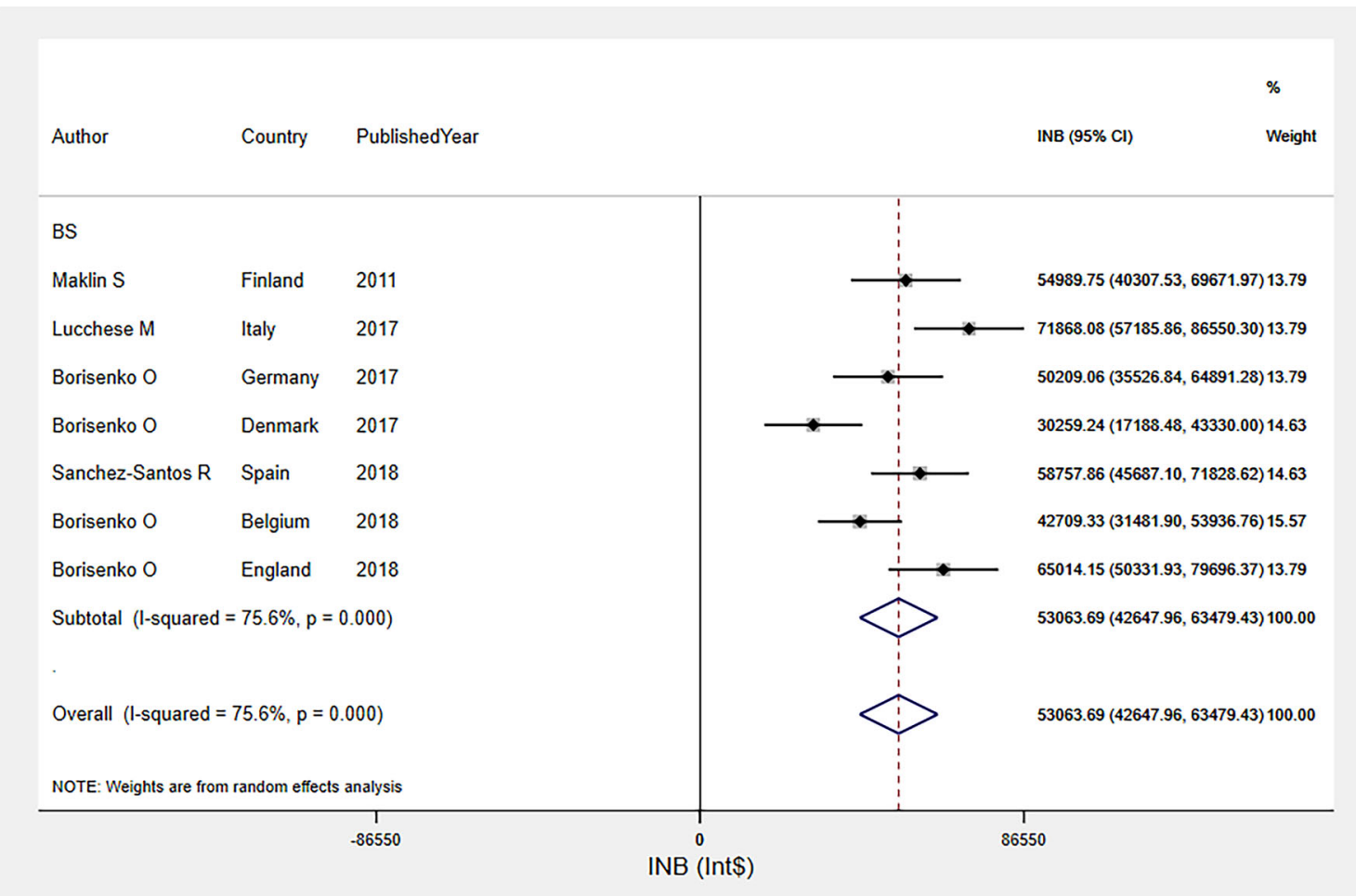

Fig. 3 Pooled INBs of bariatric surgery among mixed obesity group in high income countries over 10-year time horizon 
Pooled INBs of Bariatric Surgery among Obesity with Diabetes in HICs

The INBs of bariatric surgery among obesity with diabetes group were pooled from seven studies [33, 37, 42, 43, 47, $48,50]$ over life-time horizon (see Fig. 4). The pooled INB was $\$ 80,826.28\left(95 \%\right.$ CI $\$ 32,500.75, \$ 129,151.81 ; I^{2}=$ $85.0 \%$ ), indicating that bariatric surgery was cost-effective relative to usual care. Asymmetry was observed in funnel plot and Egger's test (coefficient $=2.51, \mathrm{SE}=0.92, p=0.041$ ) (Fig. S4). A contour-enhanced funnel plot (Fig. S5) showed that asymmetry might be caused by heterogeneity.

\section{INBs of Bariatric Surgery in UMICs}

Of the four studies from UMICs [36, 44, 51, 52], three studies $[36,44,51]$ with five comparisons were conducted in mixed obesity group, while four comparisons [36, 44, 51, 52] were conducted in obesity with diabetes group $[36,44,51,52]$ (see Table S6). Time horizon varied across studies, i.e., 5 years [51], 10 years [44], 20 years [36], and 50 years [52]. All studies adopted a payer perspective $[36,44,51,52]$. For mixed group obesity group, the evidence was inconclusive. On the other hand, all studies conducted among obesity with diabetes group revealed that bariatric surgery was costeffective with the INBs ranging from $\$ 4,015.28$ to
$\$ 40,867.97$ (see Table S6). Since studies had varying time horizons, the INBs were not pooled.

\section{Risk of Bias}

Risk of bias was assessed using ECOBIAS checklist (see Table S7). All studies had no bias in terms of comparator, outcomes measurement, and discounting. Four studies [27, $31,45,46]$ had unclear risk of wrong model bias. Three studies $[45,46,51]$ adopted short-term time-horizon of 5 years, four studies $[28,31,36,44]$ used mid-term period of 10 and 20 years, and 18 studies [25-27, 29, 30, 32-35, 37-43, 48, 49] adopted lifetime horizon. Partial biases related to treatment effect, intermittent data collection, and quality of life weight occurred in almost all studies. Data on long-term treatment effect of bariatric surgery and utility per unit of BMI change were limited. Moreover, half of the studies [25-29, 31, 37, 38, $40,44-46,48,49,51,52]$ were subjected to bias related to internal consistency.

\section{Discussion}

We conducted a systematic review and meta-analysis for pooling INBs of bariatric surgery as compared with usual care. A total of 24 and 4 studies from HICs and UMICs were included in the review, but only data from HICs were

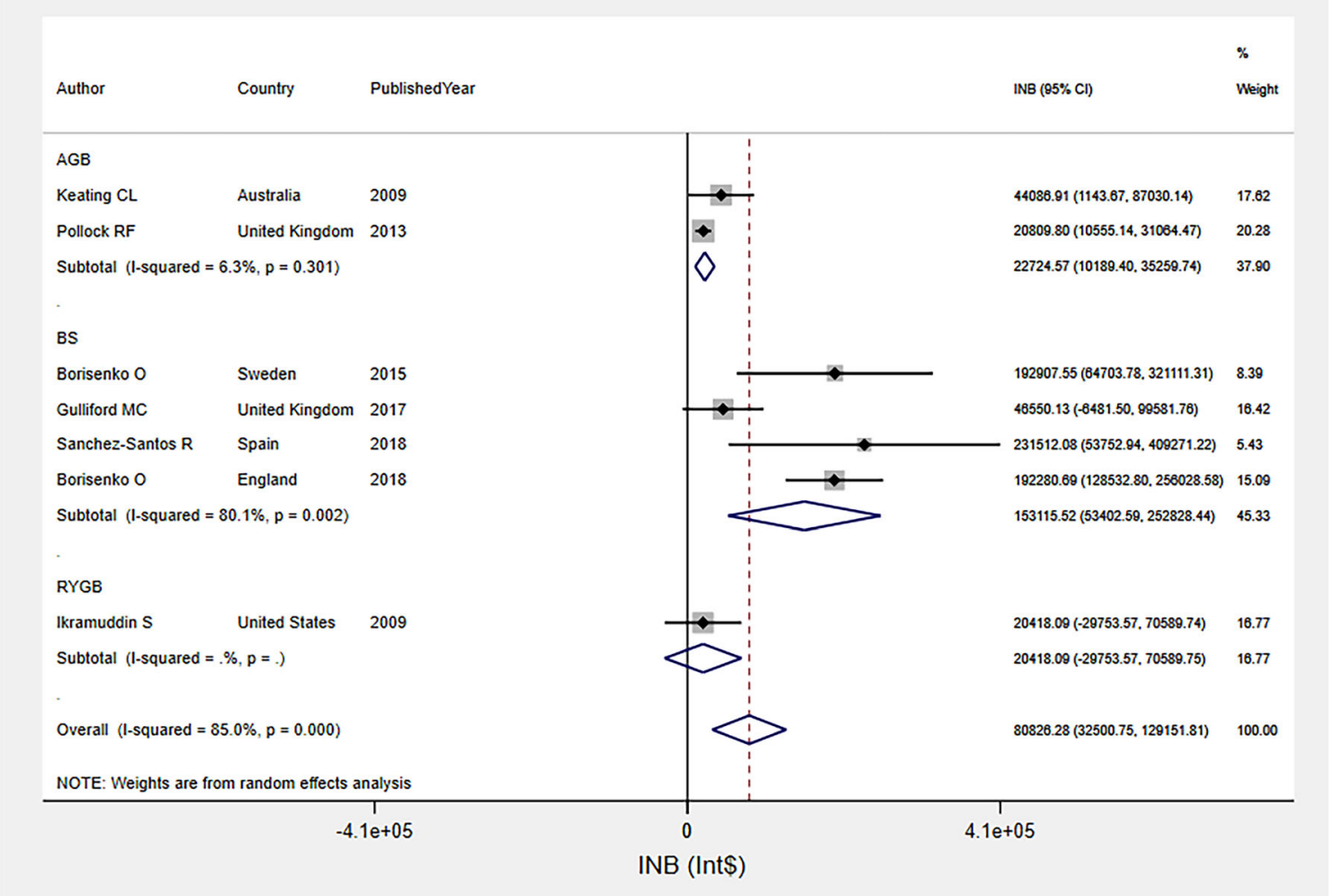

Fig. 4 Pooled INBs of bariatric surgery among obesity with diabetes group in high income countries over life-time horizon 
sufficiently pooled. We found that bariatric surgery was a cost-effective intervention for mixed obesity group (i.e., with/without diabetes) in HICs over 10-year and lifetime time horizons. In addition, it was a cost-effective intervention for patients with obesity and diabetes in both HICs and UMICs from a payer perspective. For HICs, our analysis provides economic evidences in support of the current clinical practice guideline [53], which recommends bariatric surgery for patients with $\mathrm{BMI} \geq 40 \mathrm{~kg} / \mathrm{m}^{2}$ and patients with $\mathrm{BMI} \geq 35 \mathrm{~kg}$ / $\mathrm{m}^{2}$ with co-morbidity.

Comparing the three recent systematic reviews [7, 9, 54], they included 35 unique studies, while our study included 28 studies. Of the 28 included studies, 4 [31, 44, 51, 52] were not included in the previous systematic reviews [7, 9, 54]. Eleven studies [55-65] were included in the previous studies [7, 9, $54]$ but were not eligible in our present review since they were studies among adolescents $(N=4)$ [61, 63-65], with BMI $<32$ $\mathrm{kg} / \mathrm{m}^{2}(N=2)[55,56]$, with insufficient data for calculating INB $(N=3)[57,59,62]$, and studied in a specific group of patients $(N=2)[58,60]$. Almost all studies adopted a payer perspective, which considered only direct medical costs, rather than a broader societal perspective. This might be due to the need for evidences among payers to support reimbursement decision while there had been growing demand for bariatric surgery. Notably, a study [29], which was conducted from a societal perspective, indicated that bariatric surgery was costeffective and that the direct medical costs associated with both the surgery and treatment of comorbidities represented a major cost component.

For mixed obesity group (i.e., with/without diabetes), all evidences from HICs indicated that bariatric surgery was costeffective over lifetime time horizon. Nevertheless, three studies, which adopted 2-year [27], 5-year [30], and 10-year [31] time horizons, found that bariatric surgery was not cost-effective. Our findings revealed that bariatric surgery might be cost-effective in studies employing a $\geq 10$-year time horizon and more cost-effective over lifetime horizon. This could be possibly explained by the fact that costs for bariatric surgery were driven by high cost of surgery in the first few years [66], while the benefit of significantly lower healthcare expenditures due to the reduction of comorbidities could be observed in the long term after surgery [7]. Most included studies performed bariatric surgery on patients with a mean age $\geq 40$ years. They projected all costs and outcomes incurred after the surgery throughout a lifetime period. It should be noted that INB with a lifetime horizon for these patients can be considered cost-effective as bariatric surgery can help prevent them from comorbidities and complications related to obesity, which finally can improve their health outcomes and decrease healthcare costs. In addition, performing the bariatric surgery with a lifetime horizon among patients with younger ages (i.e., 20 years) was even more cost-effective $[38,40]$. This is due to the fact that the young patients continue to gain benefits from surgery for a longer period as compared with their older counterparts.

Nevertheless, there is currently limited evidence on the long-term effects of bariatric surgery. Further studies on the long-term effectiveness and safety of bariatric surgery are warranted to improve the validity of cost-effectiveness studies.

According to our review, bariatric surgery was costeffective (i.e., positive INB) in all 14 studies (29 comparisons) conducted among obesity with diabetes group. This is similar to a previous systematic review [9], which found that almost all studies (6/7) conducted among obesity with diabetes group indicated that bariatric surgery was cost-effective. Bariatric surgery was generally believed to be more cost-effective in obesity with diabetes group than general obesity group. However, we found that the pooled INB (95\% CI) in obesity with diabetes group over lifetime horizon was lower than mixed obesity group but not significant, i.e., $\$ 80,826.28$ $(\$ 32,500.75, \$ 129,151.81)$ vs. \$101,897.96 (\$79,390.93, $\$ 124,404.99)$, respectively. It should be noted that remission was higher among patients with recent onset of diabetes and those who were not taking insulin $[67,68]$. Less benefit of bariatric surgery was reported in diabetic patients who had already developed complications [67]. Variation in such characteristics of diabetes patients in each included study might possibly lead to the unclear conclusion when comparing the benefits of bariatric surgery between mixed obesity and obesity with diabetes group. Further studies carefully designed to compare cost-effectiveness of bariatric surgery among various characteristics of patients with diabetes (i.e., early diagnosed vs. long-term diabetes) are also warranted.

Presently, many types of bariatric surgery have been used in clinical practice. Nevertheless, most included studies reported mixed types of bariatric surgery. Subgroup analysis by types of bariatric surgery was performed for AGB, SG, and RYGB. We found that SG and AGB were cost-effective when compared with usual care, but not for RYGB. This could be explained by the fact that only three studies for RYGB were included for pooling and they had very large variances. Consequently, the pooled INB has high uncertainty and the interpretation should be made with caution. Based on current evidence, it is still inconclusive on which specific types of bariatric surgery should be selected. Further studies comparing cost-effectiveness among different types of the surgery are thus warranted.

Although this study was the first to provide quantitative evidences on value for money of bariatric surgery by estimating and pooling INB of bariatric surgery, certain limitations were needed to be addressed. First, all included economic evaluation studies were performed in HICs and UMICs, but none was conducted in lower-middle-income countries (LMICs) and low-income countries (LICs). Therefore, future economic evaluation studies of bariatric surgery in such 
settings should be further investigated. Second, in some cases, where dispersion parameters had not been reported (scenario 5 ), variances of INBs were adopted from other studies, which had comparable characteristics in terms of country, country income level, characteristics of patients, types of intervention, perspective, discount rate, and time. Nevertheless, adopted dispersion might not fully represent the actual dispersion of the INBs. Third, according to the quality assessment, almost all studies had biases related to treatment effects such that evidences on long-term effects of bariatric surgery were scarce. To improve validity of cost-effectiveness evidences, studies on the long-term treatment effect of bariatric surgery are warranted. Finally, due to data availability, we could only pool the value for money of bariatric surgery among obese patients with diabetes but not with other comorbidities, such as hypertension and obstructive sleep apnea.

In conclusion, our findings indicated that bariatric surgery seems to be cost-effective over 10-year and lifetime horizons in HICs for both mixed obesity group (i.e., with/ without diabetes) and obesity with diabetes group. The pooled INB for bariatric surgery as compared with usual care in HICs was estimated to be between $\$ 81,000$ and $\$ 102,000$ over the lifetime horizon. For UMICs, bariatric surgery seemed to be cost-effective as compared with usual care among obesity with diabetes group with the INBs ranging from $\$ 4,000$ to $\$ 41,000$.

Supplementary Information The online version contains supplementary material available at https://doi.org/10.1007/s11695-021-05415-9.

Acknowledgments This work was part of training at Mahidol University Health Technology Assessment (MUHTA) program, with scholarship provided by Mahidol University and the International Decision Support Initiative (iDSI). This work was produced as part of the International Decision Support Initiative (www.idsihealth.org) which supports countries to get the best value for money from health spending. iDSI receives funding support from the Bill \& Melinda Gates Foundation, the UK Department for International Development, and the Rockefeller Foundation. This work was supported in part by the Bill \& Melinda Gates Foundation (grant number OPP1087363). Under the grant conditions of the Foundation, a Creative Commons Attribution 4.0 Generic License has already been assigned to the Author Accepted Manuscript version that might arise from this submission. The findings, interpretations and conclusions expressed in this article do not necessarily reflect the views of the aforementioned funding agencies.

Authors' contribution All authors contributed to the study conception and design. Searching: P.N. Study selection: P.N., M.T., and B.S.B. Quality assessment and data extraction: P.N., M.T., and B.S.B. Data analysis: P.N., U.C., B.S.B., A.T., and M.T. The first draft of the manuscript was written by P.N. and all authors commented on previous versions of the manuscript. All authors read and approved the final manuscript.

\section{Declarations}

Ethical approval statement This article does not contain any studies with human participants or animals performed by any of the authors.

Informed consent statement Informed consent does not apply.

Ethical statement We confirm that the manuscript has not been published elsewhere as a whole or partly and is not under consideration by another journal. Each author has contributed significantly to the work and agreed to this submission. All authors have approved the manuscript for submission

Conflicts of interest The authors declare no competing interests.

Open Access This article is licensed under a Creative Commons Attribution 4.0 International License, which permits use, sharing, adaptation, distribution and reproduction in any medium or format, as long as you give appropriate credit to the original author(s) and the source, provide a link to the Creative Commons licence, and indicate if changes were made. The images or other third party material in this article are included in the article's Creative Commons licence, unless indicated otherwise in a credit line to the material. If material is not included in the article's Creative Commons licence and your intended use is not permitted by statutory regulation or exceeds the permitted use, you will need to obtain permission directly from the copyright holder. To view a copy of this licence, visit http://creativecommons.org/licenses/by/4.0/.

\section{References}

1. Park CH, Nam SJ, Choi HS, et al. Comparative efficacy of bariatric surgery in the treatment of morbid obesity and diabetes mellitus: a systematic review and network meta-analysis. Obes Surg. 2019;29: 2180-90.

2. Gloy VL, Briel M, Bhatt DL, et al. Bariatric surgery versus nonsurgical treatment for obesity: a systematic review and metaanalysis of randomised controlled trials. BMJ. 2013;347:f5934.

3. Colquitt JL, Pickett K, Loveman E, et al. Surgery for weight loss in adults. Cochrane Database Syst Rev. 2014;(8):Cd003641.

4. Cheng J, Gao J, Shuai X, et al. The comprehensive summary of surgical versus non-surgical treatment for obesity: a systematic review and meta-analysis of randomized controlled trials. Oncotarget. 2016;7(26):39216-30.

5. Chang S-H, Stoll CRT, Song J, et al. Bariatric surgery: an updated systematic review and meta-analysis, 2003-2012. JAMA Surg. 2014;149(3):275-87.

6. Muller-Stich BP, Senft JD, Warschkow R, et al. Surgical versus medical treatment of type 2 diabetes mellitus in nonseverely obese patients: a systematic review and meta-analysis. Ann Surg. 2015;261(3):421-9.

7. Xia Q, Campbell JA, Ahmad H, et al. Bariatric surgery is a costsaving treatment for obesity - a comprehensive meta-analysis and updated systematic review of health economic evaluations of bariatric surgery. Obes Rev. 2020;21(1):e12932.

8. Lopes EC, Heineck I, Athaydes G, et al. Is bariatric surgery effective in reducing comorbidities and drug costs? A systematic review and meta-analysis. Obes Surg. 2015;25(9):1741-9. 
9. Campbell JA, Venn A, Neil A, et al. Diverse approaches to the health economic evaluation of bariatric surgery: a comprehensive systematic review. Obes Rev. 2016;17(9):850-94.

10. Reed SD. Statistical considerations in economic evaluation: a guide for cardiologists. Eur Heart J. 2014;35:1652-6.

11. Hoch JS, Dewa CS. A clinician's guide to correct cost-effectiveness analysis: think incremental not average. Can J Psychiatry. 2008;53(4):267-74.

12. McCabe C, Claxton K, Culyer AJ. The NICE cost-effectiveness threshold: what it is and what that means. Pharmacoeconomics. 2008;26(9):733-44.

13. World Health Organization. Choosing interventions that are costeffective [Internet]. Geneva: World Health Orgnanization 2014.

14. Net Monetary Benefit [Internet]. York Health Economics Consortium. 2016. Available from: https://yhec.co.uk/glossary/ net-monetary-benefit/.

15. Crespo C, Monleon A, Díaz W, et al. Comparative efficiency research (COMER): meta-analysis of cost-effectiveness studies. BMC Med Res Methodol. 2014;14(1):139.

16. Haider S, Chaikledkaew U, Thavorncharoensap M, et al. Systematic review and meta-analysis of cost-effectiveness of rotavirus vaccine in low-income and lower-middle-income countries. Open Forum Infect Dis. 2019;6(4):ofz117.

17. Bagepally BS, Gurav YK, Anothaisintawee T, et al. Cost utility of sodium-glucose cotransporter 2 inhibitors in the treatment of metformin monotherapy failed type 2 diabetes patients: a systematic review and meta-analysis. Value Health. 2019;22(12):1458-69.

18. Chaiyakittisopon K, Pattanaprateep O, Ruenroengbun N, et al. Evaluation of the cost-utility of phosphate binders as a treatment option for hyperphosphatemia in chronic kidney disease patients: a systematic review and meta-analysis of the economic evaluations. Eur J Health Econ. 2021; https://doi.org/10.1007/s10198-02101275-3.

19. Moher D, Liberati A, Tetzlaff J, et al. Preferred reporting items for systematic reviews and meta-analyses: the PRISMA statement. PLoS Med. 2009;6(7):e1000097.

20. World Economic Outlook Database [Internet]. 2019 [cited 14 November 2019]. Available from: https://www.imf.org/external/ pubs/ft/weo/2019/01/weodata/download.aspx.

21. Adarkwah CC, van Gils PF, Hiligsmann M, et al. Risk of bias in model-based economic evaluations: the ECOBIAS checklist. Expert Rev Pharmacoecon Outcomes Res. 2016;16(4):513-23.

22. World Bank Country and Lending Groups [Internet]. 2019 [cited 14 November 2019]. Available from: https://datahelpdesk.worldbank. org/knowledgebase/articles/906519-worldbank-country-andlending-groups.

23. DerSimonian R, Laird N. Meta-analysis in clinical trials. Control Clin Trials. 1986;7(3):177-88.

24. Palmer TM, Peters JL, Sutton AJ, et al. Contour-enhanced funnel plots for meta-analysis. Stata J. 2008;8(2):242-54.

25. Craig BM, Tseng DS. Cost-effectiveness of gastric bypass for severe obesity. Am J Med. 2002;113(6):491-8.

26. Campbell J, McGarry LA, Shikora SA, et al. Cost-effectiveness of laparoscopic gastric banding and bypass for morbid obesity. Am J Manag Care. 2010;16(7):e174-87.

27. McEwen LN, Coelho RB, Baumann LM, et al. The cost, quality of life impact, and cost-utility of bariatric surgery in a managed care population. Obes Surg. 2010;20(7):919-28.

28. Maklin S, Malmivaara A, Linna M, et al. Cost-utility of bariatric surgery for morbid obesity in Finland. Br J Surg. 2011;98(10): 1422-9.
29. Faria GR, Preto JR, Costa-Maia J. Gastric bypass is a cost-saving procedure: results from a comprehensive Markov model. Obes Surg. 2013;23(4):460-6.

30. Castilla I, Mar J, Valcarcel-Nazco C, et al. Cost-utility analysis of gastric bypass for severely obese patients in Spain. Obes Surg. 2014;24(12):2061-8.

31. Lewis L, Taylor M, Broom J, et al. The cost-effectiveness of the LighterLife weight management programme as an intervention for obesity in England. Clin Obes. 2014;4(3):180-8.

32. Wang BC, Wong ES, Alfonso-Cristancho R, et al. Costeffectiveness of bariatric surgical procedures for the treatment of severe obesity. Eur J Health Econ. 2014;15(3):253-63.

33. Borisenko O, Adam D, Funch-Jensen P, et al. Bariatric surgery can lead to net cost savings to health care systems: results from a comprehensive European decision analytic model. Obes Surg. 2015;25(9):1559-68.

34. Borisenko O, Mann O, Duprée A. Cost-utility analysis of bariatric surgery compared with conventional medical management in Germany: a decision analytic modeling. BMC Surg. 2017;17(1).

35. Borisenko O, Lukyanov V, Johnsen SP, Funch-Jensen P. Cost analysis of bariatric surgery in Denmark made with a decision-analytic model. Dan Med J. 2017;64(8).

36. Cohen RV, Luque A, Junqueira $\mathrm{S}$, et al. What is the impact on the healthcare system if access to bariatric surgery is delayed? Surg Obes Relat Dis. 2017;13(9):1619-27.

37. Gulliford MC, Charlton J, Prevost T, et al. Costs and outcomes of increasing access to bariatric surgery: cohort study and costeffectiveness analysis using electronic health records. Value Health. 2017;20(1):85-92.

38. James R, Salton RI, Byrnes JM, et al. Cost-utility analysis for bariatric surgery compared with usual care for the treatment of obesity in Australia. Surg Obes Relat Dis. 2017;13(12):2012-20.

39. Lucchese M, Borisenko O, Mantovani LG, et al. Cost-utility analysis of bariatric surgery in Italy: results of decision-analytic modelling. Obes Facts. 2017;10(3):261-72.

40. Alsumali A, Eguale T, Bairdain S, et al. Cost-effectiveness analysis of bariatric surgery for morbid obesity. Obes Surg. 2018;28(8): 2203-14.

41. Borisenko O, Lukyanov V, Debergh I, et al. Cost-effectiveness analysis of bariatric surgery for morbid obesity in Belgium. J Med Econ. 2018;21(4):365-73.

42. Borisenko O, Lukyanov V, Ahmed AR. Cost-utility analysis of bariatric surgery. Br J Surg. 2018;105:1328-37.

43. Sanchez-Santos R, Padin EM, Adam D, et al. Bariatric surgery versus conservative management for morbidly obese patients in Spain: a cost-effectiveness analysis. Expert Rev Pharmacoecon Outcomes Res. 2018;18(3):305-14.

44. Assumpcão RP, Bahia LR, da Rosa MQM, et al. Cost-utility of gastric bypass surgery compared to clinical treatment for severely obese with and without diabetes in the perspective of the Brazilian Public Health System. Obes Surg. 2019.

45. Ackroyd R, Mouiel J, Chevallier JM, et al. Cost-effectiveness and budget impact of obesity surgery in patients with type-2 diabetes in three European countries. Obes Surg. 2006;16(11):1488-503.

46. Anselmino M, Bammer T, Fernandez Cebrian JM, et al. Costeffectiveness and budget impact of obesity surgery in patients with type 2 diabetes in three European countries(II). Obes Surg. 2009; 19(11):1542-9.

47. Ikramuddin S, Klingman CD, Swan T, et al. Cost-effectiveness of Roux-en-Y gastric bypass in type 2 diabetes patients. Am J Manag Care. 2009;15(9):607-15. 
48. Keating CL, Dixon JB, Moodie ML, et al. Cost-effectiveness of surgically induced weight loss for the management of type 2 diabetes: modeled lifetime analysis. Diabetes Care. 2009;32(4):567-74.

49. Hoerger TJ, Zhang P, Segel JE, et al. Cost-effectiveness of bariatric surgery for severely obese adults with diabetes. Diabetes Care. 2010;33(9):1933-9.

50. Pollock RF, Muduma G, Valentine WJ. Evaluating the costeffectiveness of laparoscopic adjustable gastric banding versus standard medical management in obese patients with type 2 diabetes in the UK. Diabetes Obes Metab. 2013;15(2):121-9.

51. Gil-Rojas Y, Garzón A, Lasalvia P, et al. Cost-effectiveness of bariatric surgery compared with nonsurgical treatment in people with obesity and comorbidity in Colombia. Value Health Reg Issues. 2019;20:79-85.

52. Viratanapanu I, Romyen C, Chaivanijchaya K, et al. Costeffectiveness evaluation of bariatric surgery for morbidly obese with diabetes patients in Thailand. J Obes. 2019;2019:1-6.

53. Di Lorenzo N, Antoniou SA, Batterham RL, et al. Clinical practice guidelines of the European Association for Endoscopic Surgery (EAES) on bariatric surgery: update 2020 endorsed by IFSO-EC, EASO and ESPCOP. Surg Endosc. 2020;34(6):2332-58.

54. Alsumali A, Al-Hawag A, Samnaliev M, et al. Systematic assessment of decision analytic models for the cost-effectiveness of bariatric surgery for morbid obesity. Surg Obes Relat Dis. 2018;14(7): 1041-59.

55. Tang Q, Sun Z, Zhang N, et al. Cost-effectiveness of bariatric surgery for type 2 diabetes mellitus: a randomized controlled trial in China. Medicine. 2016;95(20):e3522.

56. Song HJ, Kwon JW, Kim YJ, et al. Bariatric surgery for the treatment of severely obese patients in South Korea-is it cost effective? Obes Surg. 2013;23(12):2058-67.

57. Salem L, Devlin A, Sullivan SD, et al. Cost-effectiveness analysis of laparoscopic gastric bypass, adjustable gastric banding, and nonoperative weight loss interventions. Surg Obes Relat Dis. 2008;4(1):26-32.

58. Neff R, Havrilesky LJ, Chino J, et al. Bariatric surgery as a means to decrease mortality in women with type I endometrial cancer - an intriguing option in a population at risk for dying of complications of metabolic syndrome. Gynecol Oncol. 2015;138(3):597-602.

59. Lee YY, Veerman JL, Barendregt JJ. The cost-effectiveness of laparoscopic adjustable gastric banding in the morbidly obese adult population of Australia. PLoS One. 2013;8(5):e64965.

60. Klebanoff MJ, Corey KE, Chhatwal J, et al. Bariatric surgery for nonalcoholic steatohepatitis: a clinical and cost-effectiveness analysis. Hepatology. 2017;65(4):1156-64.

61. Klebanoff MJ, Chhatwal J, Nudel JD, et al. Cost-effectiveness of bariatric surgery in adolescents with obesity. JAMA Surg. 2017;152(2):136-41.

62. Kim DD, Arterburn DE, Sullivan SD, et al. Economic value of greater access to bariatric procedures for patients with severe obesity and diabetes. Med Care. 2018;56(7):583-8.

63. Chang SH, Stoll CR, Colditz GA. Cost-effectiveness of bariatric surgery: should it be universally available? Maturitas. 2011;69(3): 230-8.

64. Bairdain S, Samnaliev M. Cost-effectiveness of adolescent bariatric surgery. Cureus. 2015;7(2):e248-e.

65. Ananthapavan J, Moodie M, Haby M, et al. Assessing costeffectiveness in obesity: laparoscopic adjustable gastric banding for severely obese adolescents. Surg Obes Relat Dis. 2010;6(4): 377-85.

66. Smith VA, Arterburn DE, Berkowitz TSZ, et al. Association between bariatric surgery and long-term health care expenditures among veterans with severe obesity. JAMA Surg. 2019:e193732.

67. Busetto L. Timing of bariatric surgery in people with obesity and diabetes. Ann Transl Med. 2015;3(7):94.

68. Jans A, Naslund I, Ottosson J, et al. Duration of type 2 diabetes and remission rates after bariatric surgery in Sweden 2007-2015: a registry-based cohort study. PLoS Med. 2019;16(11):e1002985.

Publisher's Note Springer Nature remains neutral with regard to jurisdictional claims in published maps and institutional affiliations. 